\title{
La réception des travaux en didactique de la langue : quelles résistances?
}

\author{
Marie-Laure Elalouf \\ Université de Cergy-Pontoise - IUFM de l'académie de Versailles \\ UMR 7114 MODyCo - CNRS - Paris X-Nanterre
}

\begin{abstract}
Cette contribution se propose d'analyser les résistances à l'évolution des contenus de l'enseignement grammatical, alors même que la labilité des acquisitions conceptuelles et terminologiques est régulièrement observée tant chez les élèves que chez les enseignants en formation. Je prendrai pour seul poste d'observation la question de la morphologie verbale qui est une zone critique sur le plan de l'apprentissage de la langue écrite et j'essaierai de montrer comment les prescriptions institutionnelles et les logiques éditoriales se confortent pour marginaliser l'examen critique des descriptions les plus courantes, en dépit des difficultés de conceptualisation qu'elles semblent générer.

Je partirai d'enquêtes récentes sur la graphie des formes verbales chez des élèves de l'enseignement obligatoire et des enseignants en formation. Je confronterai ces résultats à d'autres enquêtes sur les ouvrages de référence utilisés par des étudiants se destinant à l'enseignement. Et j'essaierai de montrer comment les logiques éditoriales ont marginalisé des tentatives visant à substituer à une description prétendument exhaustive une logique de production des formes verbales. Cette étude pose plus largement la question des démarches que peut promouvoir la didactique de la langue dans un contexte où les publications scolaires, parascolaires et tout public véhiculent des représentations de la langue très prégnantes et à fort investissement identitaire.
\end{abstract}

\section{La graphie des formes verbales : une zone critique dans la scolarité obligatoire et au-delà.}

La thèse de Catherine Brissaud (1998) a permis un état des lieux des difficultés des collégiens à partir de l'analyse de 1812 erreurs sur 10569 formes verbales relevées dans un corpus de copies. Il apparaît que les verbes du premier groupe y sont les plus fréquents et que, contrairement aux idées reçues, ils suscitent plus d'erreurs que ceux du $3^{\mathrm{e}}$ groupe aux différents niveaux de la scolarité. L'étude montre aussi que parmi les formes verbales, le participe passé et le passé simple concentrent le plus d'erreurs et que les formes en /E/ forment une zone particulièrement critique du fait de l'homophonie. Une étude transversale plus récente (2006) met en évidence une tendance à choisir la forme en -é comme alternative à l'infinitif, et à lui faire porter des marques de genre et de nombre en rapport avec le sujet de la phrase ou un argument de l'infinitif (*elles vont allées).

L'enquête publiée en 2007 par Danièle Manesse et Danielle Cogis confirme que l'orthographe grammaticale est une difficulté majeure et que la graphie des formes verbales est très inégalement maitrisée : à côté d'une forme synthétique comme ils vont, correctement écrite par 9 élèves sur 10 dès le CM2, la forme ils distribuent n'est maîtrisée que par 4 élèves sur 10. Il apparaît que la rencontre entre un radical à finale vocalique et une marque verbale sans correspondance à l'oral est une cause de difficulté qui subsiste pour un élève sur 5 en fin de $3^{\mathrm{e}}$.

L'étude d'un corpus de travaux d'étudiants de la seconde année de Licence en 1998 à leur entrée dans le métier d'enseignant en passant par la préparation du CAPES montre que des zones de difficultés subsistent au terme d'un cursus universitaire de Lettres en cas d'homophonie :

- alignement de la forme adjective du participe passé sur la forme correspondante du présent de l'indicatif (un drapeau brandit, un chevalier accomplit, elle a remplit); 
- erreur sur la marque flexionnelle quand le radical se termine par une voyelle (renvoit/ renvoie, défie/ défit, conclue/ conclut, retient/ retiend);

- hésitation entre le futur et le conditionnel (quand j'enseignerais)

- erreur sur la garphie de l'infinitif (servire, suffir);

- erreur sur les finales en [e] (lui avoir prouvez, vous pouvez utilisé, nous avons lister);

- surgénéralisation de l'accord du participe passé avec l'auxiliaire avoir pour les verbes pronominaux et intransitifs (nous nous sommes rendus compte, l'appellation a évoluée).

Une enquête menée à la même période auprès de futurs professeurs des écoles permet de constater qu'en fin de formation initiale ils sont inquiets, voire angoissés par rapport à leur mission d'enseignement de l'orthographe française, déclarant tous n'être pas suffisamment formés dans ce domaine (Péret, Sautot, Brissaud, 2008).

\section{Les outils usuels des futurs enseignants}

Ces constats conduisent à s'interroger sur les représentations de la langue que se sont forgées les futurs enseignants au cours de leurs études et à se demander si les ouvrages de référence qu'ils utilisent les confortent ou sont susceptibles de les faire évoluer. Très tôt dans la scolarité, la conjugaison se constitue en une sous-discipline dont les contours sont problématiques: elle isole le verbe des régularités morphologiques caractérisant d'autres classes de mots ; elle mêle des considérations morphologiques et syntaxiques en présentant comme des formes des constructions telles que le passif, le gérondif ou ce que la tradition appelle conditionnel passé $2^{\mathrm{e}}$ forme; elle propose des classements s'appuyant sur un découpage sans cohérence des terminaisons, ce qui conduit à multiplier les exceptions; enfin, elle véhicule une terminologie héritée de la tradition grammaticale qui induit des confusions entre temps verbaux et temps chronologique, passé et accompli. Mais si l'on interroge les futurs enseignants sur leurs outils de référence, on constate qu'ils ne sont guère en mesure de prendre un recul critique par rapport à cette présentation.

Les futurs professeurs des écoles sont très nombreux à citer le Bescherelle et le Bled, comme des ouvrages qu'ils possèdent depuis le début de leur scolarité et auxquels ils se réfèrent, les considérant comme une autorité. Plus généralement, ils sont une majorité à se référer à un manuel scolaire plutôt qu'à une grammaire actualisée, comme une première enquête menée en 1998 et renouvelée en 2007 a pu le montrer (Elalouf, 2008).

Chez les futurs professeurs de collège, la possession d'un dictionnaire est générale, alors que l'acquisition d'une grammaire de référence doit faire l'objet d'une prescription. Et c'est dans le dictionnaire que les formes verbales sont vérifiées. Or la présentation des conjugaisons dans ces ouvrages repose sur un classement en groupes qui isole deux ensembles de paradigmes présentés comme réguliers, le premier et le deuxième groupe, et un nombre très important de verbes dits irréguliers qui semblent nécessiter la mémorisation des différentes formes. Or la notion d'irrégularité recouvre des faits très divers : variation du radical, particularité orthographique non spécifique au verbe (la cédille de nous plaçons), particularité orthographique résultant de l'adjonction normale d'une désinence à la base (vérifier ou créer), désinence exceptionnelle comme le $-x$ de je veux ou le -tes de vous faites, conjugaison recourant à différents étymons. Ainsi, le Dictionnaire du français contemporain présente 85 modèles de conjugaison, le Petit Robert comme le Robert du français langue étrangèrel langue seconde 61. Certains ouvrages, comme le Bescherelle Junior et de nombreux manuels scolaires regroupent par analogie les verbes en -cer, -ger, eler, -endre, -aindre, etc. selon un découpage qui n'est ni syllabique, ni morphologique et qui illustre bien l'ambiguité du mot terminaison. En effet, il n'y a pas de solution de continuité entre ces présentations tout public et les manuels scolaires les plus répandus en France : même présentation par groupes, même organisation des tableaux de formes, même listes d'exceptions, avec en arrière plan théorique la non prise en compte de l'oral et l'absence de procédures fiables pour distinguer radical et désinence (Ahr, S. 2007). 


\section{La didactique de la morphologie verbale : une voie étroite}

La didactique de la morphologie verbale, qui s'est développée au départ sur la base des travaux de Jean Dubois, peine à diffuser dans le monde enseignant français alors qu'elle a fait sa place dans l'espace francophone : le répertoire des verbes conçu dans le cadre de la rénovation de l'enseignement vaudois en 1990 propose une progression en fonction de la fréquence des verbes et met en vis-à-vis d'une part les alternances de bases et les désinences orales et d'autre part leur correspondance à l'écrit ; plus récemment la Grammaire pédagogique du français distingue les variations du radical puis celles des terminaisons écrites. Mais il faut constater que ces ouvrages ne relèvent pas du marché éditorial, comme c'est le cas des manuels scolaires en France. Les manuels qui ont proposé une approche du système verbal tenant compte de l'oral et proposant des règles d'engendrement qui allient identification d'une base et choix de désinences ont connu une diffusion limitée et sont restés cantonnés à deux maisons d'éditions de moindre importance : Delagrave pour la série Bâtir une grammaire (1977-79) dirigée par Bernard Combettes et Grammaire pour lire et écrire de Roberte Tomassone ; Retz pour les ouvrages de Bernard Couté.

Une conjonction de facteurs concourt à un immobilisme de la présentation du système verbal en France. D'une part, le marché éditorial adapte ses productions aux pratiques les plus répandues sur la base d'études de marché et diffuse des ouvrages par ses relais dans les établissements sans que ceux-ci soient soumis à une véritable critique. D'autre part, une exploitation idéologique de l'opposition entre mémorisation et compréhension du système verbal a fait disparaître des programmes la notion de règles d'engendrement, introduite en 2002, qui n'avait connu qu'une traduction timide dans les pratiques. Seule la question de la fréquence des verbes est mieux prise en compte. Les résistances ne sont donc pas externes mais internes et il importe de les analyser pour envisager des évolutions, notamment en formation.

La première résulte de l'isolement de la classe du verbe dans la rubrique conjugaison. Rien n'invite les enseignants à mettre en relation le verbe avec d'autres classes de mots et d'autres niveaux d'analyse de la langue. Les terminaisons sont généralement présentées de façon synthétiques, ce qui masque des régularités transversales, notamment pour les marques de personnes. Les phénomènes phonétiques et orthographiques qui affectent le verbe ne sont pas mis en relation avec leur correspondant pour d'autres classes de mots (alternance vocalique : soulever/ soulèvement; allongement de la base : facil-i-tél fin-issant; lettre diacritique: nous mangeons, une mangeoire). Inversement, les catégories qui affectent spécifiquement le verbe, le temps et le mode, sont mises sur le même plan que celles qui l'affectent indirectement, la personne par le phénomène de l'accord, le nombre où l'opposition concerne surtout la $3^{\mathrm{e}}$ personne, le genre qui n'affecte que la forme adjective du verbe improprement appelée participe passé (S. Meleuc, 1999).

La seconde résistance concerne la prise en compte des formes orales. Les préventions autour de l'utilisation de l'API en classe se doublent d'une interrogation sur l'utilité d'un classement par bases. S'il s'ajoute au classement traditionnel, comme c'est le cas dans la Nouvelle grammaire du français de J. Dubois et R. Lagane qui propose un classement des formes écrites par groupes puis un classement des formes orales par bases, il peut être considéré comme une surcharge. S'il vient complexifier le classement par groupes en introduisant à l'intérieur de chaque groupe les variations par base, comme c'est le cas dans la Grammaire du français, il n'est pas nécessairement perçu comme un gain. Pour que la prise en compte des formes orales soit mise au service de règles d'engendrement, l'ordre de présentation des personnes doit être repensé. Le regroupement des personnes 1, 2, 3 et 6 d'une part, 4 et 5 d'autre part, en détachant les deux cas où les désinences portent l'accent tonique au présent de l'indicatif, simplifie la mise en relation entre formes orales et formes écrites et permet de dégager une base fondamentale sur laquelle construire les autres désinences temporelles et modales. Il devient alors possible dans la perspective d'une grammaire de production, de faire appel à la base orale pour la graphie de la partie lexicale du verbe, puis de choisir les désinences en fonction du phonème final de la base, selon des alternatives que n'envisagent pas les élèves ou les étudiants qui achoppent sur la graphie de distribuent ou revoit.

Mais si ce type de calcul reste difficile, c'est aussi parce que les enseignants ne sont guère sensibilisés aux phénomènes phonétiques à la jonction du radical et de la désinence, comme l'effacement de la voyelle 
thématique devant une désinence à initiale vocalique et son maintien dans les autres cas. Si quelques formes de passé simple monosyllabiques requièrent une explication historique, beaucoup d'irrégularités sont explicables en synchronie si l'on tient compte de cette alternance, comme le montre Hélène Huot (2005). Seul ce type d'analyse permet d'ailleurs de rendre compte de la particularité morphologique de la forme adjective du verbe qui ne comporte pas de désinence mais seulement un élargissement vocalique (en $-\dot{e},-i,-u$ ) ou consonantique (en $-s$ ou $-t$ ), ce qui autorise la suffixation (posé-ment, écrit-ure) et l'adjonction d'un morphème de genre ou de nombre.

Enfin, une dernière résistance repose sur la facilité présumée de certains paradigmes par rapport à d'autres. Le présent de l'indicatif est généralement abordé en premier parce qu'il est jugé plus simple sur le plan conceptuel, alors que sur le plan morphologique, il ne comporte pas de marques de temps ni de mode et que ses marques de personnes ne sont audibles qu'aux personnes 4 et 5 . L'imparfait, dont la base est régulière ainsi que les marques de temps et de personnes est plus propice à des manipulations de commutation comme l'a montré Carole Tisset (2005).

$\mathrm{Si}$ au lieu de partir de l'écrit pour procéder au découpage radical/ terminaison, on se place dans la perspective de l'élève qui doit encoder une forme verbale, on peut s'appuyer sur sa connaissance de la base orale, identifier les verbes dont le radical se termine par une voyelle thématique au présent de l'indicatif aux personnes $1,2,3,6$ comme à infinitif $(e+r)$, signaler les très rares cas où la base se termine par une lettre muette comme prendre. Le choix des désinences de mode et de temps faisant appel à des marques orales, seules celles de personnes comportent des morphogrammes qui exigent une attention particulière. Mais pour accompagner efficacement cet encodage des formes verbales, l'enseignant doit avoir une vue d'ensemble du système orthographique du français et pouvoir expliquer la polyvalence $\mathrm{du}-\mathrm{e}$ (voyelle thématique dans $i l$ joue, marque du subjonctif alternant avec $-i$ dans $q u$ 'il conclue, marque de féminin dans une affaire conclue), comme celle du $-s$ ou du $-t$. Et c'est sans doute un dernier point d'achoppement, compte tenu des présentations les plus répandues de l'orthographe.

Pourtant, une telle approche est nécessaire au développement de procédures morphosyntaxiques pour la graphie des formes verbales : identifier les marques, les associer à des catégories grammaticales entrant dans des constructions syntaxiques analysables, distinguer ce qui relève du système morphologique du verbe et de celui du nom. Ajoutons que dans une perspective d'éveil à la diversité des langues, observer l'alternance des bases permet d'éclairer d'autres systèmes verbaux, tandis que l'identification des morphèmes de mode, de temps et de personnes permet d'établir des équivalences que la notion floue de terminaison ne permet pas de faire. L'enjeu dépasse donc la seule maîtrise du français.

Les tâches de la formation sont donc multiples et complexes. Pour comprendre les représentations que les futurs enseignants ont du système verbal, les aider à maîtriser les zones qui restent problématiques, il importe de connaître leurs outils de référence habituels, de les engager à une réflexion sur leur conception et leurs limites, à partir des difficultés qu'ils rencontrent, de les doter de procédures pour les utiliser autrement et faire naître si possible le besoin de consulter d'autres ressources. Il s'agit en même temps de développer chez le futur enseignant la capacité d'expliquer ses choix à des élèves qui ont connu d'autres approches, aux parents, à l'équipe pédagogique. Sur ce point, la didactique de la discipline s'articule étroitement à la question des pratiques professionnelles que la formation doit penser conjointement.

\section{Bibliographie}

Ahr, D. (2007). Le verbe dans douze manuels de $6^{\mathrm{e}}$. Bulletin d'information sur les manuels scolaires $n^{\circ} 71$.

Brissaud, C. \& Bessonnat, D (2001). L'orthographe au collège, pour une autre approche. Grenoble: CRDPDelagrave.

Denis, D. \& Sancier-Château, A. (1994). Grammaire du français. Paris : livre de poche.

Dubois, J. \& Lagane, R (1973, 1989, $2^{\mathrm{e}}$ ed.). Nouvelle grammaire du français. Paris :Larousse. 
ISBN 978-2-7598-0358-3, Paris, 2008, Institut de Linguistique Française

Didactique et enseignement, français langue maternelle, français langue seconde DOI $10.1051 / \mathrm{cmlf08316}$

Elalouf, M.-L. (2008, à paraître). Des outils effectifs des futurs enseignants à la convocation des savoirs linguistiques et réciproquement. In Caoail, E. \& Gourdet P., La formation des enseignants du premier degré : quelles articulations entre savoirs scientifiques et pratiques quotidiennes? Namur : Presses universitaires.

Huot, H. (2005). Morphologie. Forme et sens des mots du français. Paris : Armand Colin.

Jaffré, J.-P. \& Brissaud, C. (2006). Homophonie et hétérographie, un point nodal de l'orthographe. In HonvaultDucrocq, R. L'orthographe en question. Presses universitaires de Rouen et du Havre, collection Dyalang : 145168.

Manesse, D. \& Cogis, D. (2007). Orthographe, à qui la faute ?. Paris : ESF.

S. Meleuc, (1999). Didactique de la conjugaison. Le verbe « autrement ». Paris : Bertrand-Lacoste.

Péret, C., Sautot, J.-P. et Brissaud C. (2008). Les professeurs des écoles entrant dans le métier et la norme orthographique. In Pellat, J.-C., Brissaud, C. et Jaffré, J.-P. (dir.), Nouvelles recherches en orthographe. Limoges, éditions Lambert Lucas.

Tisset, C. (2005). Observer, manipuler, enseigner la langue au cylce 3. Paris : Hachette. 